\title{
Vessel-Selective 4D-MRA Using Superselective Pseudocontinuous Arterial Spin-Labeling with Keyhole and View-Sharing for Visualizing Intracranial Dural AVFs
}

\author{
(D) O. Togao, (D) M. Obara, (D). Kikuchi, (D). Helle, (D). Arimura, (D) A. Nishimura, (D). Wada, (D). Murazaki, \\ (D) M. Van Cauteren, (D) A. Hiwatashi, and (D) K. Ishigami
}

\begin{abstract}
BACKGROUND AND PURPOSE: An accurate assessment of the hemodynamics of an intracranial dural AVF is necessary for treatment planning. We aimed to investigate the utility of 4D-MRA based on superselective pseudocontinuous arterial spin-labeling with CENTRA-keyhole and view-sharing (4D-S-PACK) for the vessel-selective visualization of intracranial dural AVFs.
\end{abstract}

MATERIALS AND METHODS: We retrospectively analyzed the images of 21 patients 12 men and 9 women; mean age, 62.2 [SD,19.2] years) with intracranial dural AVFs, each of whom was imaged with DSA, 4D-S-PACK, and nonselective 4D-MRA based on pseudocontinuous arterial spin-labeling combined with CENTRA-keyhole and view-sharing (4D-PACK). The shunt location, venous drainage patterns, feeding artery identification, and Borden classification were evaluated by 2 observers using both MRA methods on separate occasions. Vessel selectivity was evaluated on 4D-S-PACK.

RESULTS: Shunt locations were correctly evaluated in all 21 patients by both observers on both MRA methods. With 4D-S-PACK, observers 1 and 2 detected $76(80.0 \%, P<.001)$ and $73(76.8 \%, P<.001)$ feeding arteries of the 95 feeding arteries identified on DSA but only 39 (41.1\%) and 46 (48.4\%) feeding arteries with nonselective 4D-PACK, respectively. Both observers correctly identified 10 of the 11 patients with cortical venous reflux confirmed by DSA with both 4D-S-PACK and 4D-PACK (sensitivity $=90.9 \%$, specificity $=90.9 \%$ for each method), and they made accurate Borden classifications in 20 of the 21 patients (95.2\%) on both MRA methods. Of the 84 vessel territories examined, vessel selectivity was graded 3 or 4 in $73(91.2 \%)$ and $66(88.0 \%)$ territories by observers 1 and 2, respectively.

CONCLUSIONS: 4D-S-PACK is useful for the identification of feeding arteries and accurate classifications of intracranial dural AVFs and can be a useful noninvasive clinical tool.

ABBREVIATIONS: ASL = arterial spin-labeling; CNR = contrast-to-noise ratio; CVR = cortical venous reflux; 4D-PACK $=4 \mathrm{D}$-MRA based on pseudocontinuous arterial spin-labeling combined with CENTRA-keyhole and view-sharing; 4D-S-PACK = 4D-MRA based on superselective pseudocontinuous arterial spinlabeling with CENTRA-keyhole and view-sharing; DAVF = dural AVF; ECA = external carotid artery; PCASL = pseudocontinuous arterial spin-labeling

D ural AVFs (DAVFs) are abnormal vascular connections between arteries and veins within the dural leaflet. Intracranial DAVFs can cause a variety of clinical symptoms related to venous

Received September 22, 2021; accepted after revision December 11.

From the Departments of Molecular Imaging \& Diagnosis (O.T.), Clinical Radiology (K.K., A.H., K.I.), and Neurosurgery (K.A., A.N.), Graduate School of Medical Sciences, Kyushu University, Fukuoka, Japan; Philips Japan (M.O., M.V.C.), Tokyo, Japan; Philips Research (M.H.), Hamburg, Germany; and Division of Radiology (T.W., H.M.), Department of Medical Technology, Kyushu University Hospital, Fukuoka, Japan.

This study was supported by the Japan Society for the Promotion of Science KAKENHI grant No. JP20K08111.

Please address correspondence to Akio Hiwatashi, MD, Department of Clinical Radiology, Graduate School of Medical Sciences, Kyushu University, 3-1-1 Maidashi Higashi-ku, Fukuoka 812-8582, Japan; e-mail: hiwatashi.akio.428@m.kyushu-u.ac.jp

- Indicates open access to non-subscribers at www.ajnr.org

Indicates article with online supplemental data.

Indicates article with supplemental online videos.

http://dx.doi.org/10.3174/ajnr.A7426 congestion as well as intracranial hemorrhage. In a longitudinal study of patients with DAVFs with persistent cortical venous reflux (CVR) after treatment, the annual mortality rate was $10.4 \%$, the annual risk of intracranial hemorrhage was $8.1 \%$, and the annual risk of a nonhemorrhagic neurologic deficit was $6.9 \%{ }^{1}$ An accurate assessment of the hemodynamics of an intracranial DAVF is necessary to prevent hemorrhage, reduce intraoperative complications, and contribute to a positive clinical outcome. For optimal medical care, an efficient vascular imaging method with high diagnostic performance must be established.

The current clinical standard for the diagnosis and posttreatment follow-up of intracranial DVAFs is DSA. However, a DSA examination is an invasive medical procedure and is associated with the risk of neurologic complications and adverse effects from contrast media. Since the early 2000s, the evaluation of DAVFs using time-resolved contrast-enhanced MRA has been reported, and its usefulness in assessing the main arterial feeding 
artery, shunt site, and venous outflow in DAVFs has been shown. ${ }^{2-7}$ However, the low temporal resolution of this technique, typically $1-5$ seconds, might limit the ability to evaluate the dynamics of the inflow from the feeding arteries into the shunt in detail. A noninvasive technique that does not require contrast agents, ie, MRA using arterial spin-labeling (ASL), was developed for intracranial vascular diseases. ${ }^{8-14}$ Our group has proposed a newer imaging technique, 4D-MRA based on pseudocontinuous arterial spin-labeling combined with CENTRA-keyhole and view-sharing (4D-PACK). ${ }^{13,15}$ The acquisition times are accelerated with $4 \mathrm{D}$-PACK with the use of view-sharing and the CENTRA-keyhole technique, and visualizing the distal cerebral arteries is possible even at longer transit times. ${ }^{13}$ A drawback of the $4 \mathrm{D}$-PACK technique is that it lacks vessel selectivity, ie, the ability to provide vessel-specific visualization. ${ }^{16}$ Vessel selectivity is necessary to accurately identify the relevant vessels in each case, and this identification contributes to the decision as to whether to use DSA and to the planning of interventional treatments and surgical procedures. Several recent studies tested the ability of 4D-MRA to visualize vessel-selective flows. ${ }^{17-20}$

The superselective pseudocontinuous arterial spin-labeling (pCASL) technique with single vessel-selective labeling was originally developed for regional cerebral perfusion imaging. ${ }^{21,22}$ Our group has also developed a 4D-PACK method that uses superselective pCASL as a single-vessel-selective 4D-MRA technique, 4D-MRA based on superselective pseudocontinuous arterial spin-labeling with CENTRA-keyhole and view-sharing (4D-SPACK). ${ }^{23,24}$ Our 2020 study revealed the usefulness of $4 \mathrm{D}-\mathrm{S}$ PACK for the visualization of brain arteriovenous malformations, which are usually supplied by branches of the ICA. ${ }^{24}$ To the best of our knowledge, no study to date has used vessel-selective 4DMRA to visualize intracranial DAVFs, which are primarily supplied by branches of the external carotid artery (ECA). We conducted the present study to assess the ability of 4D-S-PACK to visualize intracranial DAVFs compared with the corresponding ability of nonselective 4D-PACK, using DSA as a reference standard.

\section{MATERIALS AND METHODS}

This retrospective investigation was approved by the institutional review board of Kyushu University Hospital (No. 2019-367). The informed consent requirement was waived on the basis of the retrospective study design. Three authors (M.O., M.H, and M.V.C.) were employees of Philips Healthcare and provided technical support for the sequence development but were not involved in the study design or interpretation of the data. The institutional authors who were not Philips Healthcare employees were responsible for handling of all data.

\section{Patients}

Our hospital's routine MR imaging protocol for intracranial arteriovenous shunt diseases has included $4 \mathrm{D}$-ASL-based MRA since June 2018. Herein, we analyzed the imaging findings of the 24 consecutive patients with intracranial DAVFs or suspected DAVFs that were identified between June 2018 and June 2021. The exclusion criteria for this study were as follows: A DSA examination was not performed $(n=0)$; examinations by MRA and DSA were not performed for the patient within the required interval of 1 month $(n=0)$; DSA revealed no DAVFs $(n=2)$; severe motion artifacts were observed in any of the images $(n=1)$; and new hemorrhages or other neurologic events appeared during the observation period $(n=0)$.

\section{MRA}

A 3T scanner (Ingenia 3.0TX; Philips Healthcare) was used for the MRA examination of all patients. The detailed principles of the $4 \mathrm{D}$-S-PACK technique have been described. ${ }^{23,24}$ In the present patient series, single-vessel labeling of the right and left ICAs and the right and left ECAs was performed for each patient (4 vessels per patient) using $4 \mathrm{D}$-S-PACK. The labeling focus was placed in the proximal portions of the ICA or ECA (Fig 1) within $3 \mathrm{~cm}$ of the bifurcation of the common carotid artery. For the labeling of each artery, $0.75 \mathrm{mT} / \mathrm{m} / \mathrm{ms}$ was set as the gradient moment for superselective pCASL in the right-to-left direction and the anterior-to-posterior direction to create a circular (approximately 2-cm diameter) labeling spot.

The label durations of 100, 200, 500, 800, 1200, 1600, and 2000 $\mathrm{ms}$ were used to obtain the images, with the following imaging parameters: $3 \mathrm{D}$ T1 turbo field echo; TR/TE $=5.0 / 1.8 \mathrm{~ms}$; flip angle $=$ $11^{\circ}$; echo-train length $=60$; slab thickness $=120 \mathrm{~mm}$; voxel size $=$ $1.0 \times 1.4 \times 1.6 \mathrm{~mm}$; and sensitivity-encoding factor $=3.0$. In each case, the keyhole had been set to $70 \%$, with $36.4 \%$ as the size of the central region. Both peripheral regions were thus $16.8 \%$ of the total $k$-space samples. The use of view-sharing further accelerated the scans; the acquisition time was 5 minutes 0 seconds for each artery. It took a total of 20 minutes to image the right and left ECAs and ICAs.

With the non-vessel-selective 4D-PACK technique, the images were obtained with $100,200,400,600,800,1200,1600$, and $2200 \mathrm{~ms}$ as the label durations. The rest of the parameters were the same as the parameters used with the $4 \mathrm{D}-\mathrm{S}-\mathrm{PACK}$ method. The acquisition time was 6 minutes 5 seconds.

DSA. For each patient's DSA examination, a standard protocol was used on a biplane multipurpose system (Artis zee; Siemens). After the injection of a 4- to $10-\mathrm{mL}$ and 3- to 6-mL bolus of iodinated contrast agent iopamidol (Iopamiro; Bayer HealthCare Pharmaceuticals) at $2-5 \mathrm{~mL} / \mathrm{s}$ and $1-3 \mathrm{~mL} / \mathrm{s}$ in the ICA and ECA, respectively, frontal and lateral views were obtained. DSA was the criterion standard examination. The neuroradiologist and neurointerventional surgeons referred to the clinical reports and discussed whether the written imaging findings were correct and whether there were additional findings to determine the final DSA findings.

DSA was the criterion standard examination, and its findings were determined on the basis of the consensus of the neuroradiologists and neurointerventional surgeons.

\section{Image Evaluation}

Observer Test. The observer tests were independently conducted by 2 board-certified radiologists (O.T. with 21 years' and K.K. with 13 years' experience). They each participated in 2 reading sessions at a 1-month interval. The observer test comprised 

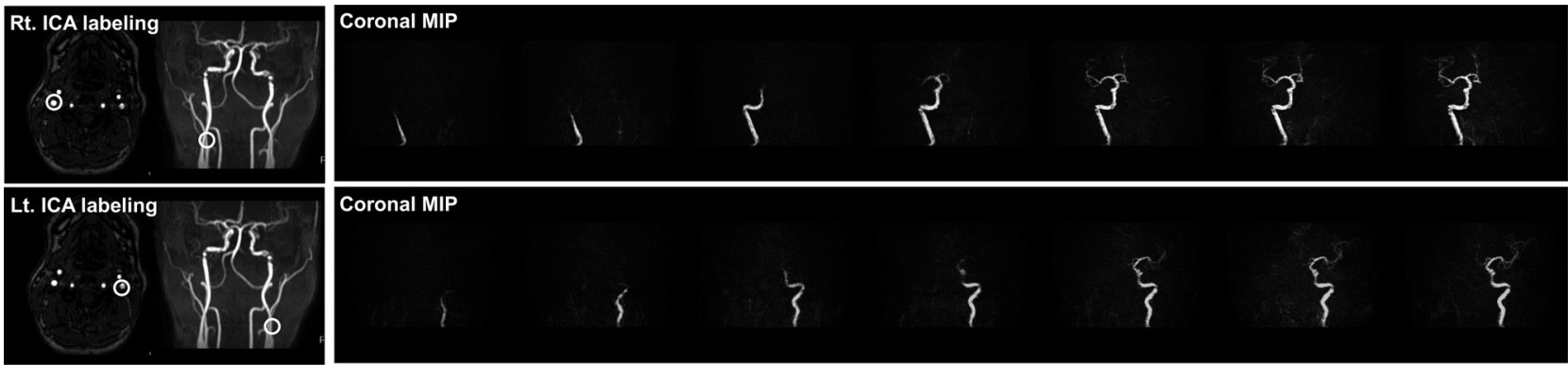

Coronal MIP
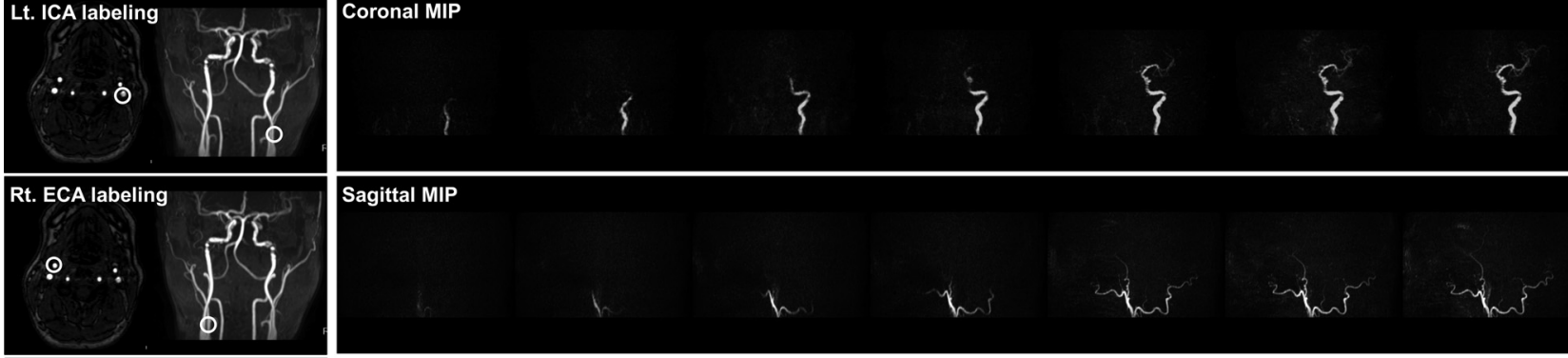

Sagittal MIP

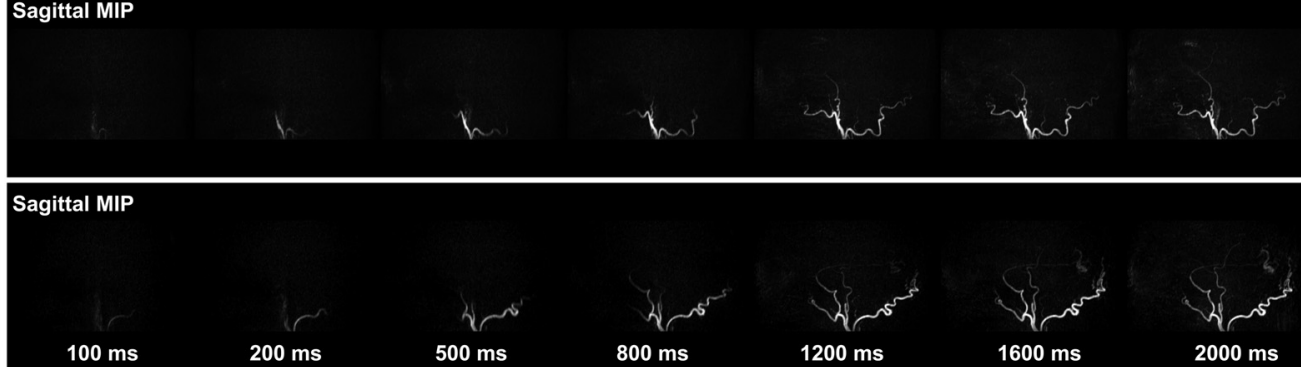

FIG 1. The locations of labeling spots. The labeling focus is placed in the proximal portions of the ICA and ECA within $3 \mathrm{~cm}$ of the bifurcation of the common carotid artery. For the labeling of each artery, $0.75 \mathrm{mT} / \mathrm{m} / \mathrm{ms}$ is set as the gradient moment for superselective pCASL in both the right-to-left and anterior-to-posterior directions, creating a circular labeling spot with an approximately 2-cm diameter. Rt. indicates right; Lt., left.

images that were obtained with either $4 \mathrm{D}$-PACK or 4D-S-PACK. A 20.8-inch liquid crystal display monitor of a PACS displayed a single $4 \mathrm{D}$-MRA image (4D-PACK or $4 \mathrm{D}-\mathrm{S}$-PACK) at a time (images other than those of the selected series were not shown). The observer evaluated only full MIP images from either type of 4D-MRA. The observer was tasked with identifying the shunt location (eg, transverse-sigmoid sinus, the cavernous sinus, anterior skull base, or superior sagittal sinus) and the feeding arteries, the presence/absence of a CVR; and the Borden classification, which is 1 of 3 types: type I, venous drainage directly into the dural venous sinus; type II, venous drainage into the dural venous sinus with a CVR; and type III, venous drainage that is directly into cortical veins (only when a CVR was present).

Vessel Selectivity. When the patient's 4D-S-PACK images were used, the observer assessed the vessel selectivity using a grading system that has been described. ${ }^{18,24}$ Briefly, the system uses a score of 1 for clearly depicted unlabeled vessels; 2 for partially depicted unlabeled vessels, which influence the interpretation of the images and the diagnosis; 3 for slightly depicted, unlabeled vessels with no influence on the diagnosis; and 4 for complete selectivity of vessels, with almost no visualization of unlabeled vessels. The score was not recorded if the image quality was judged poor and nondiagnostic due to a low SNR ratio or image artifacts.

Quantitative Assessment of the Visualization of the Method. In each patient's case, the contrast-to-noise ratio (CNR) was obtained for both feeding arteries and draining veins by a board-certified neuroradiologist (O.T., with 21 years' experience). Three circular ROIs (typically $50-70 \mathrm{~mm}^{2}$ ) were first set on each of the vessels (ie, the feeding arteries and draining veins) and on the background stationary tissues of the brain parenchyma. The patient's DSA images were used for the placement of 3 ROIs, and the locations of the
ROIs on both types of MRA were matched with those on the DSA images. The following equation was used to determine the vesselto-stationary tissue CNRs:

$$
\mathrm{CNR}=\left(\text { Vessel }_{\max }-\mathrm{ST}_{\mathrm{ave}}\right) / \mathrm{ST}_{\mathrm{SD}},
$$

where Vessel $_{\max }$ is the maximum signal intensity in the ROIs. ${ }^{13}$ $\mathrm{The} \mathrm{ST}_{\text {ave }}$ is the mean signal intensity, and $\mathrm{ST}_{\mathrm{SD}}$ is the $\mathrm{SD}$ in the stationary-tissue ROI.

\section{Statistical Analyses}

We used the $\chi^{2}$ test to compare the ability of DSA, 4D-PACK, and $4 \mathrm{D}-\mathrm{S}-\mathrm{PACK}$ to identify the shunt location, the presence/ absence of CVR, and the Borden classification. The paired $t$ test was used to compare the number of correctly identified feeding arteries between $4 \mathrm{D}-\mathrm{PACK}$ and $4 \mathrm{D}-\mathrm{S}$-PACK. The levels of intermodality agreement (between DSA and 4D-S-PACK or $4 \mathrm{D}$-PACK) concerning the number of feeding arteries were determined by calculating the weighted- $\kappa$ coefficient $(\kappa<$ 0.20 , poor; $0.21-0.40$, fair; $\kappa=0.41-0.60$, moderate; $\kappa=0.61-$ 0.80 , good; $\kappa=0.81-0.90$, very good; $\kappa>0.90$, excellent agreement). We also compared the CNRs at the 100, 200, 400, 800,1200 , and $1600 \mathrm{~ms}$ time points between the 4D-PACK and $4 \mathrm{D}-\mathrm{S}$-PACK using the paired $t$ test. (These time points were chosen because they are common to both MR imaging methods.) All of the statistical analyses were performed with GraphPad Prism 8.4.3 (GraphPad Software). P values $<.05$ were significant.

\section{RESULTS}

As summarized in Table 1, we analyzed the images of 21 patients (12 men, 9 women; 62.2 [SD, 19.2] years of age; median, 70.0 years; range, $19-85$ years). An interval of 3.6 (SD, 4.4) days passed between the patients' DSA and $4 \mathrm{D}-\mathrm{MR}$ imaging examinations. 
Table 1: Patient clinical characteristics

\begin{tabular}{lcllrl}
\hline Patient No. & Age & Sex & Shunt Location & Borden Classification & Symptom or Event \\
\hline 1 & 19 & M & R CS & Red eye \\
2 & 22 & F & L TS & Headache \\
3 & 27 & F & R CS & Visual loss, red eye \\
4 & 51 & M & L ASB & III & Headache \\
5 & 51 & M & R CS & I & Free \\
6 & 60 & M & R TS & Headache, tinnitus \\
7 & 61 & F & L TS & III & Loss of consciousness, intracranial hemorrhage \\
8 & 62 & M & L TS & III & Vertigo, double vision \\
9 & 63 & M & R TS & II & Tinnitus \\
10 & 65 & M & L TS & I & Tinnitus \\
11 & 70 & F & R TS & I & Headache, wamble \\
12 & 71 & M & L CS & II & Free \\
13 & 71 & M & R TS & III & Paresthesia of the right arm \\
14 & 72 & F & L ASB & I & Visual field abnormality \\
15 & 73 & F & R CS & I & Tinnitus \\
16 & 75 & F & L TS & II & Tinnitus \\
17 & 76 & F & L TS & I & Tinnitus \\
18 & 77 & F & R TS & III & Vertigo, tinnitus \\
19 & 77 & M & SSS & I & Visual loss \\
20 & 78 & M & R TS & Headache, intracranial hemorrhage \\
21 & 85 & M & L TS & III & Hee \\
\hline
\end{tabular}

Note:-R indicates right; L, left; TS, transverse sigmoid sinus; CS, cavernous sinus; ASB, anterior skull base; SSS, superior sagittal sinus.

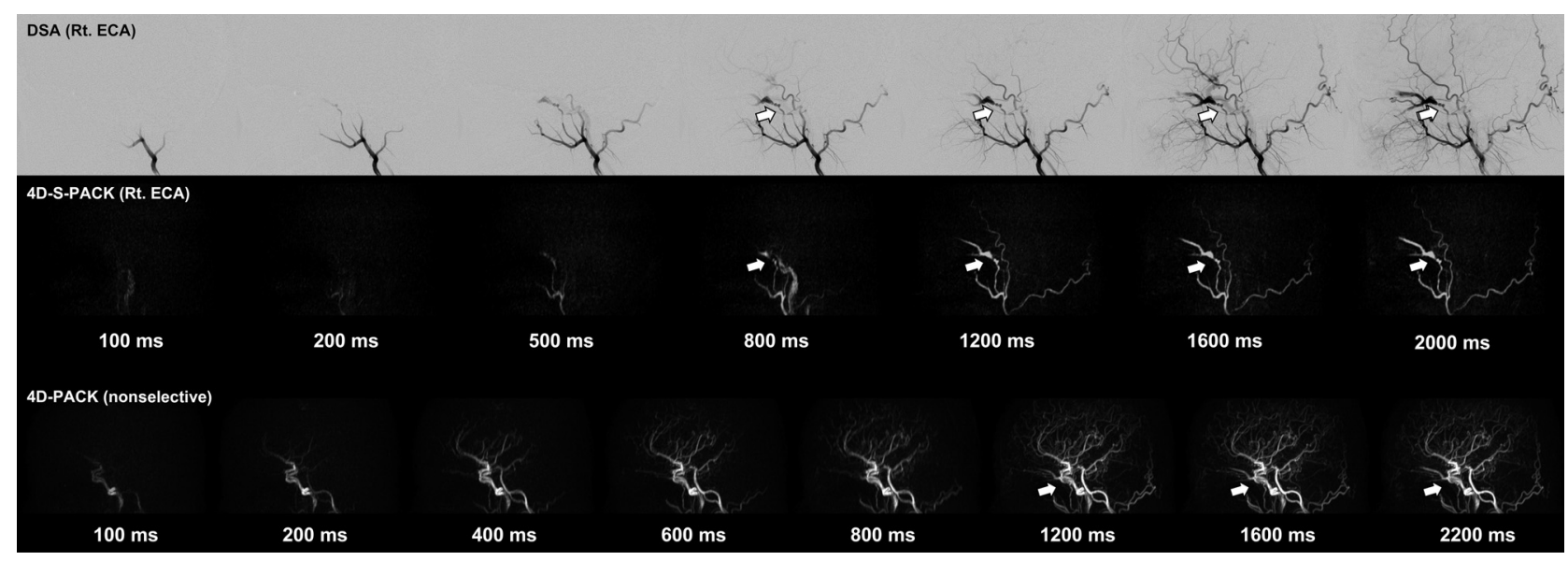

FIG 2. A 27-year-old woman with an intracranial DAVF at the cavernous sinus that is supplied by the right accessory meningeal artery (white arrow). The 4D-S-PACK (middle row) labeling of the right ECA clearly depicts the feeding artery (accessory meningeal artery) and draining veins (cavernous sinus and ophthalmic veins) as seen on the patient's DSA (upper row). In contrast, it is difficult to identify the feeding artery on 4DPACK (lower row) because of the overlap of many other unrelated vessels. Rt. indicates right.

The shunt locations were the cavernous sinus ( $n=5$ patients), the transverse-sigmoid sinus $(n=13)$, the superior sagittal sinus $(n=1)$, and the anterior skull base $(n=2)$. A total of 95 feeding arteries were identified in the 21 patients on DSA. CVR was present in 11 patients. The Borden classifications were I $(n=10$ patients), II ( $n=5)$, and III $(n=6)$.

\section{Observer Test}

Both 4D-PACK and 4D-S-PACK correctly identified the shunt locations in all 21 patients by both observers $(P>.99)$. The Online Supplemental Data summarize the identification of the feeding artery on both MRA techniques. Of the 95 feeding arteries identified on DSA, observer 1 detected 76 (80.0\%, $P<.001)$ with 4 D-S-PACK and $39(41.1 \%)$ with 4 D-PACK. Observer 2 detected 73 feeding arteries $(76.8 \%, P<.001)$ with
4D-S-PACK and $46(48.4 \%)$ with 4D-PACK. Intermodality agreement for observer 1 between DSA and 4D-S-PACK and between DSA and 4D-PACK was fair $(\kappa=0.60)$ and poor $(\kappa=$ $0.10)$, respectively. Intermodality agreement for observer 2 between DSA and 4D-S-PACK and between DSA and 4D-PACK was fair $(\kappa=0.54)$ and poor $(\kappa=0.18)$, respectively.

Both observers correctly identified 10 of the 11 patients with CVR with both 4D-S-PACK and 4D-PACK (sensitivity $=90.9 \%$, specificity $=90.9 \%$ for each method, $P>.99$ ) and provided the correct Borden classification for 20 of the 21 patients (95.2\%) with both MRA methods $(P>.99)$. Neither of the 2 observers was able to identify 1 patient with reflux into the vein of Labbe with either $4 \mathrm{D}-\mathrm{PACK}$ or $4 \mathrm{D}-\mathrm{S}-\mathrm{PACK}$.

Figure 2 and the Online Supplemental Data show the case of a patient with a DAVF at the cavernous sinus supplied by the right accessory meningeal artery. With the nonselective 4D-PACK, it 

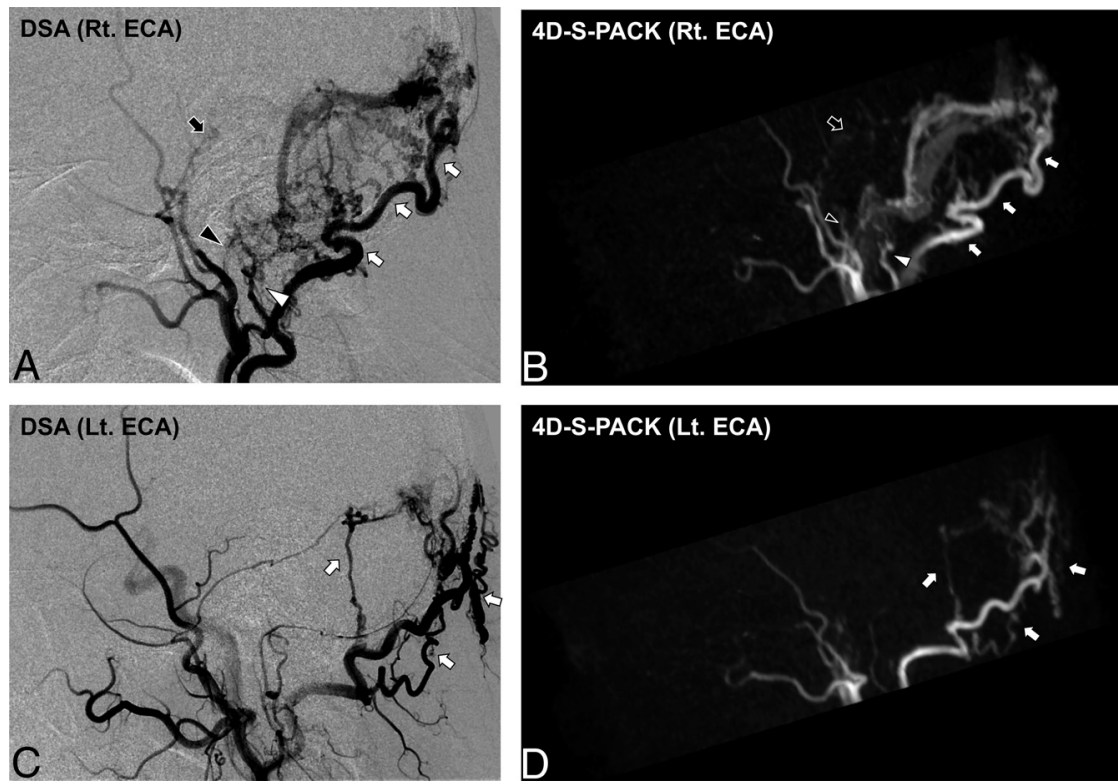

FIG 3. A 78-year-old man with an intracranial DAVF (Borden type I) at the transverse sigmoid sinus. DSA images $(A$ and $C)$ demonstrate that this shunt was supplied by multiple branches of bilateral ECAs (white arrow, occipital artery; white arrowhead, posterior auricular artery; black arrowhead, ascending pharyngeal artery; black arrow, middle meningeal artery). The 4D-S-PACK ( $B$ and $D)$ is able to selectively image the right and left ECAs at a level comparable with that of DSA, helping to accurately identify the multiple feeding arteries. Note that no ICAs or their branches are visualized on 4D-S-PACK when the selective labeling of an ECA is performed. Rt. indicates right; Lt., left.
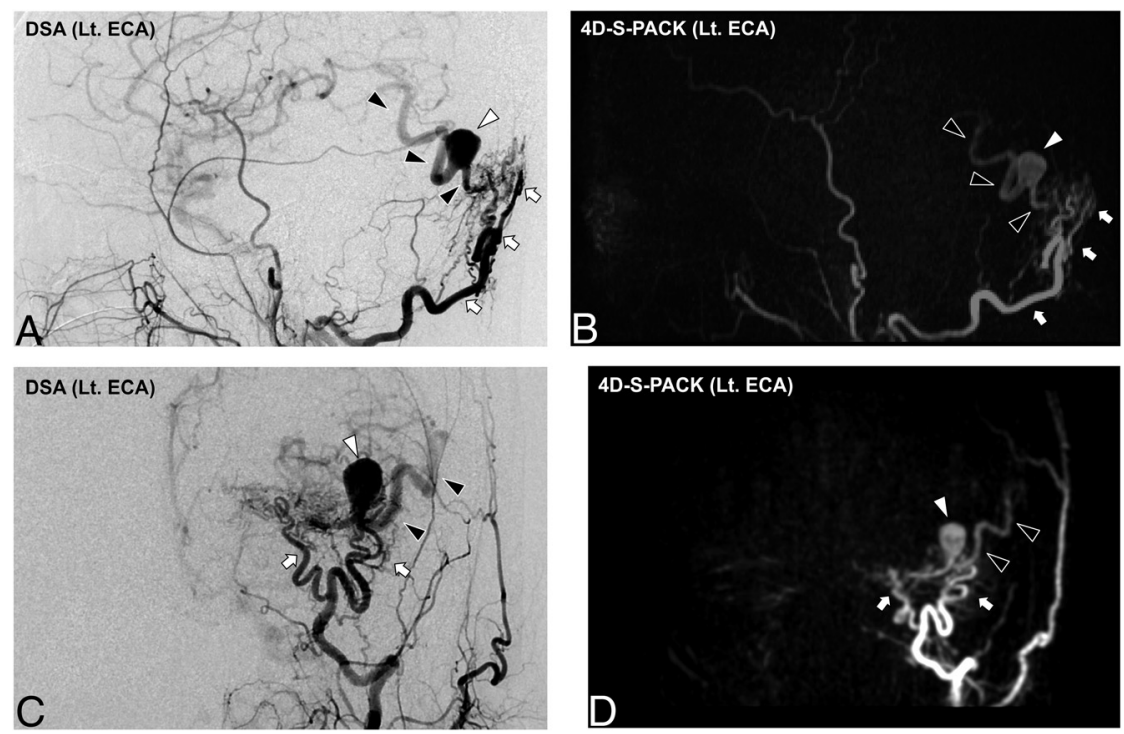

FIG 4. An 85-year-old man with an intracranial DAVF (Borden type III). DSA images (A and C) reveal that this shunt is supplied by the occipital artery branches (white arrows) at the transverse sigmoid sinus with venous drainage directly into cortical veins (black arrowheads). A varix formation (white arrowhead) is observed. In 4D-S-PACK ( $B$ and D), the left ECA is selectively imaged and the feeding artery and CVR are delineated as seen in DSA. Lt. indicates left. case of a patient with a DAVF at the transverse sigmoid sinus supplied by bilateral ECAs (Borden type I). The 4D-S-PACK was able to selectively depict the right and left ECAs as in DSA, which prevents feeding arteries from overlapping with other unrelated vessels, and thus helped to accurately identify the multiple feeding arteries. Figure 4 and the Online Supplemental Data show the case of a patient with a DAVF in the transverse sigmoid sinus with venous drainage directly into cortical veins (Borden type III). With 4D-S-PACK, the left ECA was selectively imaged and the feeding artery and CVR could be depicted with visualization comparable with that of DSA. Figure 5 and the Online Supplemental Data show the case of a patient with a DAVF in the anterior skull base, which was supplied by the left ophthalmic artery, with venous drainage directly into cortical veins and then the superior sagittal sinus (Borden type III). With 4D-S-PACK, the left ICA was selectively labeled, and the feeding artery and CVR were clearly visualized.

\section{Vessel Selectivity}

Of the 84 vessel territories examined, observer 1 graded the vessel selectivity as 4 in 68 vessel territories $(81.0 \%)$, as 3 in 9 vessel territories (10.7\%), and as 2 or 1 in none of the territories. The image quality was judged as nondiagnostic due to a low SNR ratio of a vessel or image artifacts in 7 of the $84(8.3 \%)$ territories. Of the 84 vessel territories, observer 2 graded the vessel selectivity as 4 in 60 vessel territories (71.4\%), 3 in 14 vessel territories $(16.7 \%)$, and 2 in 3 vessel territories (3.5\%). The image quality was judged as nondiagnostic due to a low SNR of the vessel or image artifacts in the same 7 of the 84 $(8.3 \%)$ territories.

\section{CNRs of Related Vessels}

The CNRs of feeding arteries and was sometimes difficult to identify the feeding artery because it overlapped with other unrelated vessels, but $4 \mathrm{D}-\mathrm{S}$-PACK was able to clearly delineate the feeding arteries as in DSA in many cases. Figure 3 and the Online Supplemental Data show the draining veins are shown in Table 2. The CNRs of feeding arteries obtained with 4D-S-PACK were significantly lower than those obtained with $4 \mathrm{D}-\mathrm{PACK}$ at $800 \mathrm{~ms}(P=.02), 1200 \mathrm{~ms}(P=.03)$, and $1600 \mathrm{~ms}(P=.006)$. The CNRs of draining veins on $4 \mathrm{D}-\mathrm{S}$ - 


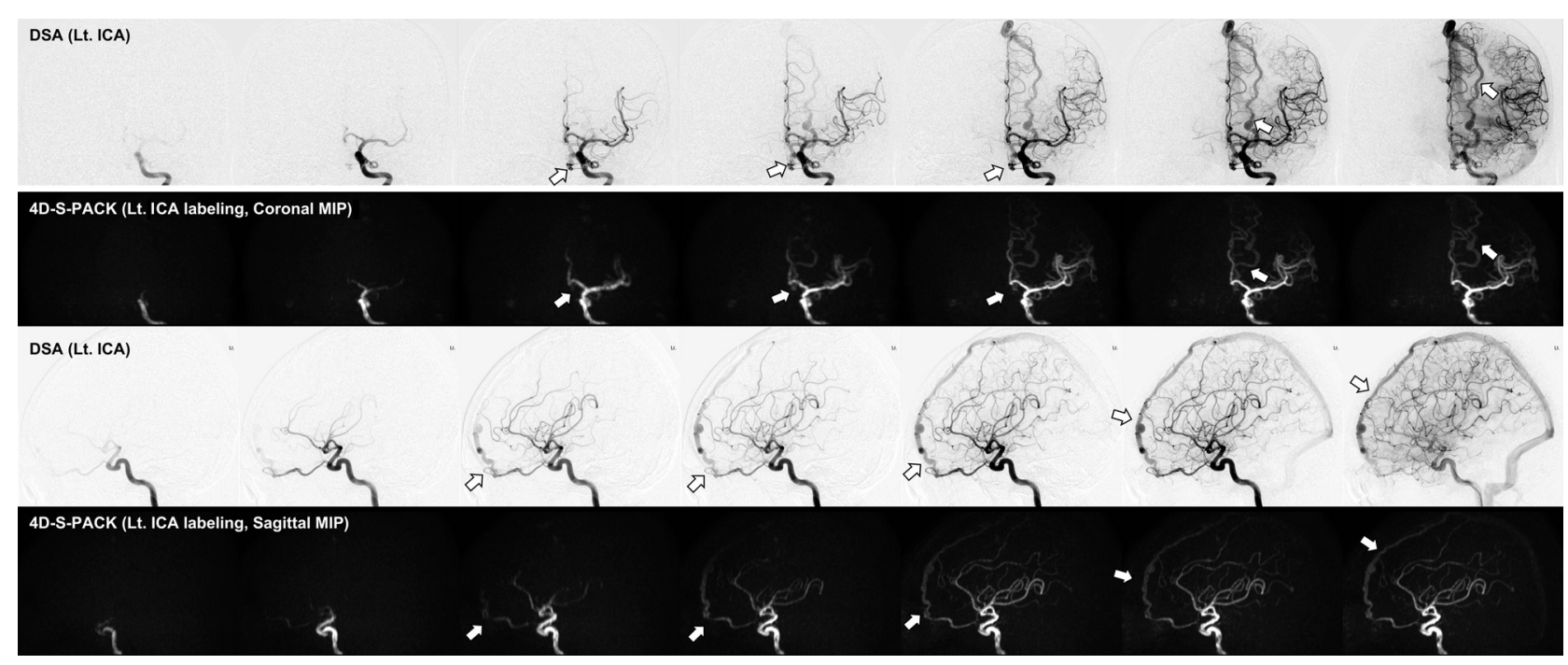

FIG 5. A 72-year-old woman with an intracranial DAVF (Borden type III) at the anterior skull base supplied by the left ophthalmic artery, with venous drainage directly into cortical veins and then the superior sagittal sinus (white arrow). In 4D-S-PACK, the left ICA is selectively imaged, and the feeding artery and CVR could be depicted. Lt. indicates left.

Table 2: CNR of related vessels ${ }^{\mathrm{a}}$

\begin{tabular}{|c|c|c|c|c|c|c|}
\hline \multirow[b]{2}{*}{ Label Duration (ms) } & \multicolumn{3}{|c|}{ Feeding Artery } & \multicolumn{3}{|c|}{ Draining Vein } \\
\hline & 4D-PACK & 4D-S-PACK & $P$ Value & 4D-PACK & 4D-S-PACK & $P$ Value \\
\hline 100 & 13.6 (SD, 12.1) & 13.7 (SD, 12.6) & .88 & $4.4(S D, 2.6)$ & $5.0(S D, 3.2)$ & .19 \\
\hline 200 & $26.0(S D, 22.3)$ & 23.1 (SD, 18.5 & .18 & 7.5 (SD, 6.1) & $6.8(S D, 5.1)$ & .41 \\
\hline 400 & 41.1 (SD, 26.4) & & & 15.4 (SD, 13.0) & & \\
\hline 500 & & 37.8 (SD, 25.9) & & & $16.3(S D, 14.0)$ & \\
\hline 600 & 49.9 (SD, 24.4) & & & 24.7 (SD, 19.6) & & \\
\hline 800 & 53.5 (SD, 24.6) & 47.1 (SD, 24.8) & .02 & 33.1 (SD, 23.9) & 26.0 (SD, 18.7) & .02 \\
\hline 1200 & $64.2(S D, 21.9)$ & $57.2(S D, 22.1)$ & .03 & 54.7 (SD, 24.0) & 39.6 (SD, 21.4) & $<.001$ \\
\hline 1600 & 66.7 (SD, 21.1) & 57.8 (SD, 21.5) & .006 & $64.2(\mathrm{SD}, 23.1)$ & $45.6(\mathrm{SD}, 20.4)$ & $<.001$ \\
\hline 2000 & & $59.2(\mathrm{SD}, 21.4)$ & & & 47.3 (SD, 20.1) & \\
\hline 2200 & 68.1 (SD, 21.0) & & & 69.7 (SD, 22.4) & & \\
\hline
\end{tabular}

Note:-4D-PACK indicates 4D-MR angiography based on pseudo-continuous arterial spin labeling combined with CENTRA-keyhole and view-sharing; 4D-S-PACK, 4D-MR angiography based on super-selective PCASL with CENTRA-keyhole and view-sharing.

${ }^{a}$ Data are expressed as mean values.

PACK were significantly lower than those on $4 \mathrm{D}-\mathrm{PACK}$ at $800 \mathrm{~ms}$ $(P=.02), 1200 \mathrm{~ms}(P<.001)$, and $1600 \mathrm{~ms}(P<.001)$.

\section{DISCUSSION}

The shunt locations of all 21 patients were correctly identified with both 4D-S-PACK and 4D-PACK. However, both observers detected a significantly greater number of feeding arteries with 4D-S-PACK compared with the nonselective 4D-PACK. Both observers correctly identified nearly all of the patients with CVR and made accurate Borden classifications using either of these MRA methods. Both observers judged that the vessel selectivity was good in most vessel territories.

Previous studies using time-resolved contrast-enhanced MRA to evaluate DAVFs have shown a relatively high performance in diagnosing the presence of a shunt, identifying the site, and classifying venous outflow. ${ }^{2-4}$ This technique may be suitable for the evaluation of venous outflow because it allows the observation of hemodynamics for a long time (30-60 seconds) after contrast administration, an advantage over ASL-based MRA. Bink et $\mathrm{al}^{5}$ evaluated DAVFs using time-resolved contrast-enhanced MRA with a resolution of 1.01 seconds and reported that the accurate identification of feeding arteries was performed correctly by $1 \mathrm{ob}-$ server in $75 \%$, but low results were obtained in the other 2 observers (35\% and 44\%). Detection of feeding arteries other than the occipital artery by the readers was not good. Nishimura et $\mathrm{al}^{6}$ evaluated DAVFs using time-resolved contrast-enhanced MRA with a temporal resolution of 1.9 seconds and found that the main feeding artery was detected correctly most often by the readers, but they did not evaluate other feeding arteries. On the other hand, in 4D-PACK and 4D-S-PACK used in our study, the temporal resolution was as high as $100-200 \mathrm{~ms}$ in the early time points, which is an advantage over time-resolved contrastenhanced MRA. In particular, 4D-S-PACK allowed us to identify multiple feeding arteries other than the main feeding arteries by separately labeling the artery.

To date, only 2 studies have used nonselective ASL-based 4DMRA to visualize intracranial DAVFs. Iryo et $\mathrm{al}^{9}$ used the pulsed ASL-based technique and reported good agreement between 4DMRA and DSA in the visualization of intracranial DAVFs. In 
contrast to our present investigation, they evaluated only 1 main feeding artery in each patient and detected 7 of 9 main feeding arteries in 9 patients. Similarly, Jang et $\mathrm{al}^{25}$ used the pulsed ASLbased technique and reported that the main feeding artery was identified in 6 of 9 patients with DAVF. Their results were consistent with ours in that 4D-PACK was able to detect most of the main feeding arteries (eg, in 19 of the 21 patients for observer 1). However, in our study, 4D-S-PACK could delineate not only the main feeding artery but also many other feeding arteries, allowing the observers to detect a significantly greater number of feeding arteries than with $4 \mathrm{D}-\mathrm{PACK}$.

The utility of $4 \mathrm{D}$-S-PACK in vessel-selective visualization of brain arteriovenous malformations has been described; ${ }^{24}$ the sensitivity of 4D-S-PACK for identifying feeding arteries of arteriovenous malformations was $100 \%$ in that study. The sensitivity of $100 \%$ in this study was likely because only the main feeding arteries of the AVMs were assessed, and not their smaller branches. In the present study, the sensitivity for detecting feeding arteries of intracranial DAVFs was lower at $80.0 \%$ and $76.8 \%$ for observers 1 and 2, respectively. A potential reason for this discrepancy is that it is more difficult to accurately label the ECA than the ICA; moreover, the branches of an ECA other than the occipital artery are often smaller than the cerebral arteries. Nevertheless, the 2 present observers could detect approximately twice as many feeding arteries with $4 \mathrm{D}-\mathrm{S}$-PACK as with $4 \mathrm{D}$ PACK. We found that in the MIP images, multiple arteries overlapped and it was difficult to identify these arteries on 4D-PACK. In contrast, $4 \mathrm{D}-\mathrm{S}-\mathrm{PACK}$ was able to differentiate these feeding arteries from other unrelated vessels. A clear visualization of the flow in the ECA territory and a suppressed visualization of the flow in the ICA territory would be clinically effective because this would improve the identification of the relevant vessels.

In studies that used nonselective pulsed ASL-based 4D-MRA to visualize cerebral arteriovenous malformations, the visualization of draining veins was worse than that of feeding arteries, ${ }^{26-28}$ suggesting that the signal of the labeled blood decreases during the image acquisitions at multiple time points after the pulsed ASL. The use of pCASL in 4D-PACK was demonstrated to provide better blood flow visualization with a long transit time. ${ }^{13,15}$ This method would, therefore, improve the visualization of draining veins in arteriovenous shunt diseases. The results of our present analyses demonstrated that this ability to visualize venous drainage was preserved in $4 \mathrm{D}$-S-PACK.

We also observed that compared with the 4D-PACK results, the CNRs of the patients' feeding arteries and draining veins were slightly lower at longer time points when 4D-S-PACK was used. This finding is similar to the results of our earlier investigation; ${ }^{24}$ it could be due to the smaller amount of blood labeled by the superselective labeling. The signal loss in 4D-S-PACK does not seem to be a significant problem in clinical practice, however, because in the observer study, the feeding arteries were better identified with $4 \mathrm{D}-\mathrm{S}-\mathrm{PACK}$ and the visibility of the draining veins was maintained.

Our assessment of vessel selectivity revealed that the targeted circulation was depicted in most patients. This indicates that the $0.75-\mathrm{mT} / \mathrm{m}$ gradient moment of superselective pCASL is efficient for selectively and separately labeling the ICA and ECA.
However, in some of our patients, unlabeled arteries were faintly visualized. In those patients, the ICA that was contralateral to the labeled ICA or the ECA was faintly visible at some time points. This could be due to the sidebands of the selective labeling. In other patients, the ipsilateral ICA or ECA was faintly visualized when the ECA or ICA, respectively, was labeled. This finding may be because the nontarget artery was partially included in the labeling area; thus, it could not be completely separated. Superselective pCASL requires careful planning of the labeling area, which increases the acquisition time. In clinical practice, the efficient training of operators is important. In the future, highly automated planning of labeling areas using artificial intelligence, for which a method has already been reported, is expected. ${ }^{29}$

There are several study limitations to address. The number of patients $(n=21)$ was small. The use of $4 \mathrm{D}-\mathrm{S}$-PACK provided good visualization of the DAVFs, but its effectiveness must be validated in larger patient series and at multiple institutions. In addition, superselective labeling targets a single artery; thus, the labeling spot is small and easily affected by patient movement. If the patient moves between labeling and imaging, the selectivity of the vessel is impaired. In this study, a poor delineation of vessels was noted in several patients, which was most likely due to patient motion. We did not evaluate false-positives in age- and sex-matched healthy subjects. However, in principle, there are no false-positives for DAVFs in ASL because veins are never visualized in healthy subjects. The labeled blood does not reach the vein at the label duration of $2000 \mathrm{~ms}$ in $4 \mathrm{D}-\mathrm{PACK}$ and $4 \mathrm{D}-\mathrm{S}$ PACK. In our study of healthy subjects ${ }^{15,23,30}$ and Moyamoya disease, ${ }^{13}$ we did not observe any veins on $4 \mathrm{D}$-PACK and $4 \mathrm{D}-\mathrm{S}$ PACK. Similarly, in our study of patients with AVMs, unrelated veins were not delineated on $4 \mathrm{D}-\mathrm{S}$-PACK. ${ }^{24}$

We did not evaluate source images of $4 \mathrm{D}-\mathrm{MRA}$ in this study to avoid complexity. In actual clinical practice, it is not realistic to review a total of 525 and 600 images of 75 slices, 7 time points (4D-S-PACK), and 8 time points (4D-PACK). However, detailed observation of the source image may improve the diagnostic performance, especially the identification of feeding arteries. Finally, it took a total of 20 minutes to image all arteries in each patient. This total acquisition time is within the time range that can be used in clinical practice, but in the future, it is necessary to shorten the time by incorporating technologies such as compressed sensing.

\section{CONCLUSIONS}

The use of the 4D-S-PACK technique provided good vessel selectivity in patients with intracranial DAVFs and helped identify the feeding arteries. The detection of CVR was mostly reliably possible. The CNRs obtained with 4D-S-PACK were slightly lower than those obtained with $4 \mathrm{D}$-PACK due to the loss of labeling by the superselective labeling, but this difference is acceptable because the vessel visualization was preserved. The $4 \mathrm{D}-\mathrm{S}$-PACK technique could thus become a widely used noninvasive clinical tool for the assessments of intracranial DAVFs.

Disclosure forms provided by the authors are available with the full text and PDF of this article at www.ajnr.org. 


\section{REFERENCES}

1. van Dijk JM, terBrugge KG, Willinsky RA, et al. Clinical course of cranial dural arteriovenous fistulas with long-term persistent cortical venous reflux. Stroke 2002;33:1233-36 CrossRef Medline

2. Meckel S, Maier M, Ruiz DS, et al. MR angiography of dural arteriovenous fistulas: diagnosis and follow-up after treatment using a time-resolved 3D contrast-enhanced technique. AJNR Am J Neuroradiol 2007;28:877-84 Medline

3. Akiba H, Tamakawa M, Hyodoh $\mathrm{H}$, et al. Assessment of dural arteriovenous fistulas of the cavernous sinuses on $3 \mathrm{D}$ dynamic MR angiography. AJNR Am J Neuroradiol 2008;29:1652-57 CrossRef Medline

4. Farb RI, Agid R, Willinsky RA, et al. Cranial dural arteriovenous fistula: diagnosis and classification with time-resolved MR angiography at 3T. AJNR Am J Neuroradiol 2009;30:1546-51 CrossRef Medline

5. Bink A, Berkefeld J, Wagner M, et al. Detection and grading of dAVF: prospects and limitations of 3T MRI. Eur Radiology 2012;22:429-38 CrossRef Medline

6. Nishimura S, Hirai T, Sasao A, et al. Evaluation of dural arteriovenous fistulas with $4 \mathrm{D}$ contrast-enhanced MR angiography at $3 \mathrm{~T}$. AJNR Am J Neuroradiol 2010;31:80-85 CrossRef Medline

7. Noguchi K, Melhem ER, Kanazawa T, et al. Intracranial dural arteriovenous fistulas: evaluation with combined 3D time-of-flight MR angiography and MR digital subtraction angiography. AJR Am J Roentgenol 2004;182:183-90 CrossRef Medline

8. Uchino H, Ito M, Fujima $\mathrm{N}$, et al. A novel application of fourdimensional magnetic resonance angiography using an arterial spin labeling technique for noninvasive diagnosis of Moyamoya disease. Clin Neurol Neurosurg 2015;137:105-11 CrossRef Medline

9. Iryo Y, Hirai T, Kai Y, et al. Intracranial dural arteriovenous fistulas: evaluation with 3-T four-dimensional MR angiography using arterial spin labeling. Radiology 2014;271:193-99 CrossRef Medline

10. Iryo $Y$, Hirai $T$, Nakamura $M$, et al. Collateral circulation via the circle of Willis in patients with carotid artery steno-occlusive disease: evaluation on 3-T 4D MRA using arterial spin labelling. Clin Radiol 2015;70:960-65 CrossRef Medline

11. Iryo $Y$, Hirai T, Nakamura $M$, et al. Evaluation of intracranial arteriovenous malformations with four-dimensional arterial-spin labeling-based 3-T magnetic resonance angiography. J Comput Assist Tomogr 2016;40:290-96 CrossRef Medline

12. Togao O, Hiwatashi A, Obara M, et al. Acceleration-selective arterial spin-labeling MR angiography used to visualize distal cerebral arteries and collateral vessels in Moyamoya disease. Radiology 2018;286:611-21 CrossRef Medline

13. Togao O, Hiwatashi A, Obara M, et al. 4D ASL-based MR angiography for visualization of distal arteries and leptomeningeal collateral vessels in moyamoya disease: a comparison of techniques. Eur Radiol 2018;28:4871-81 CrossRef Medline

14. Togao O, Hiwatashi A, Yamashita K, et al. Acceleration-selective arterial spin labeling MR angiography for visualization of brain arteriovenous malformations. Neuroradiology 2019;61:979-89 CrossRef Medline

15. Obara M, Togao O, Beck GM, et al. Non-contrast enhanced 4D intracranial MR angiography based on pseudo-continuous arterial spin labeling with the keyhole and view-sharing technique. Magn Reson Med 2018;80:719-25 CrossRef Medline

16. Suzuki Y, Fujima N, van Osch MJP. Intracranial 3D and 4D MR angiography using arterial spin labeling: technical considerations. Magn Reson Med Sci 2020;19:294-309 CrossRef Medline
17. Lindner T, Jensen-Kondering $\mathrm{U}$, van Osch MJ, et al. 3D timeresolved vessel-selective angiography based on pseudo-continuous arterial spin labeling. Magn Reson Imaging 2015;33:840-46 CrossRef Medline

18. Fujima N, Osanai T, Shimizu Y, et al. Utility of noncontrastenhanced time-resolved four-dimensional MR angiography with a vessel-selective technique for intracranial arteriovenous malformations. J Magn Reson Imaging 2016;44:834-45 CrossRef Medline

19. Okell TW, Schmitt $\mathrm{P}, \mathrm{Bi}$ X, et al. Optimization of $4 \mathrm{D}$ vessel-selective arterial spin labeling angiography using balanced steady-state free precession and vessel-encoding. NMR Biomed 2016;29:776-86 CrossRef Medline

20. Suzuki Y, Okell TW, Fujima N, et al. Acceleration of vessel-selective dynamic MR angiography by pseudocontinuous arterial spin labeling in combination with Acquisition of ConTRol and labEled images in the Same Shot (ACTRESS). Magn Reson Med 2019;81:2995-3006 CrossRef Medline

21. Helle M, Norris DG, Rufer S, et al. Superselective pseudocontinuous arterial spin labeling. Magn Reson Med 2010;64:777-86 CrossRef Medline

22. Lindner T, Austein F, Jansen O, et al. Self-controlled super-selective arterial spin labelling. Eur Radiol 2018;28:1227-33 CrossRef Medline

23. Obara M, Togao $\mathrm{O}$, Helle $\mathrm{M}$, et al. Improved selective visualization of internal and external carotid artery in 4D-MR angiography based on super-selective pseudo-continuous arterial spin labeling combined with CENTRA-keyhole and view-sharing (4D-S-PACK). Magn Reson Imaging 2020;73:15-22 CrossRef Medline

24. Togao O, Obara M, Helle M, et al. Vessel-selective 4D-MR angiography using super-selective pseudo-continuous arterial spin labeling may be a useful tool for assessing brain AVM hemodynamics. Eur Radiol 2020;30:6452-63 CrossRef Medline

25. Jang J, Schmitt P, Kim BY, et al. Non-contrast-enhanced 4D MR angiography with STAR spin labeling and variable flip angle sampling: a feasibility study for the assessment of dural arteriovenous fistula. Neuroradiology 2014;56:305-14 CrossRef Medline

26. Xu J, Shi D, Chen C, et al. Noncontrast-enhanced four-dimensional MR angiography for the evaluation of cerebral arteriovenous malformation: a preliminary trial. J Magn Reson Imaging 2011;34:1199_ 1205 CrossRef Medline

27. Yu S, Yan L, Yao Y, et al. Noncontrast dynamic MRA in intracranial arteriovenous malformation (AVM), comparison with time of flight (TOF) and digital subtraction angiography (DSA). Magn Reson Imaging 2012;30:869-77 CrossRef Medline

28. Raoult H, Bannier E, Robert B, et al. Time-resolved spin-labeled MR angiography for the depiction of cerebral arteriovenous malformations: a comparison of techniques. Radiology 2014;271:524-33 CrossRef Medline

29. Helle M, Wenzel F, van de Ven K, et al. Advanced automatic planning for super-selective arterial spin labeling flow territory mapping. In: Proceedings of the Annual Meeting of the International Society of Magnetic Resonance in Medicine, Paris, France. June 16-21, 2018;26:302

30. Murazaki H, Wada T, Togao O, et al. Optimization of 4D-MR angiography based on superselective pseudo-continuous arterial spin labeling combined with CENTRA-keyhole and view-sharing (4DS-PACK) for vessel-selective visualization of the internal carotid artery and vertebrobasilar artery systems. Magn Reson Imaging 2022;85:287-96 CrossRef Medline 